\title{
Development of Low Temperature ESEM: Exploring Sublimation
}

\author{
D. Waller, D.J. Stokes \& A.M. Donald
}

Biological \& Soft Systems, Cavendish Laboratory, Madingley Road, Cambridge, CB3 0HE, UK

Low temperature ESEM has the potential to expand the range of experiments carried out in this versatile instrument. We are exploring methodologies for the control and characterisation of specimens over a broad sub-zero temperature range and under different environmental conditions. The aim is to carry out in situ dynamic experiments as well as studies of temperature dependent changes in systems with sub-zero glass transition temperatures.

An important aspect of low temperature ESEM is control of chamber environment, which consequently affects specimen stability. For example we have made a systematic study of ice sublimation as functions of temperature and pressure, using a modified Gatan Alto 2100 cryosystem mounted in an FEI XL30 ESEM FEG. The experimental set up for low temperature ESEM can be seen in Figure 1.

First we studied sublimation rate as a function of sample temperature. The sublimation rate was calculated by measuring the change in diameter of small beads embedded in ice as it sublimed. In this experiment a constant pressure of 0.8 torr of nitrogen was used throughout, whilst the sample temperature was varied between $-90^{\circ} \mathrm{C}$ and $-55^{\circ} \mathrm{C}$. The experimental results are compared to the theoretical sublimation rate calculated from Tabor [1] in Figure 2. Both show an exponential relationship between temperature and sublimation rate, although the significant offset in temperature between experiment and theory is due to a departure from the ideal gas law.

For conventional high vacuum cryo SEM, it is generally found that sublimation of ice begins at around $-100{ }^{\circ} \mathrm{C}[2]$. However in low vacuum, using nitrogen, we find this to be nearer $-75^{\circ} \mathrm{C}$.

One explanation for this difference is the interaction of sublimated water vapour molecules with each other and with nitrogen gas present inside the chamber. This turns out to be a significant factor, leading to our second investigation: the effect of chamber pressure on sublimation rate. For example recent work has shown that at $-55^{\circ} \mathrm{C}$ the sublimation rate decreases from $0.35 \mu \mathrm{m} / \mathrm{s}$ to $0.1 \mu \mathrm{m} / \mathrm{s}$ on increasing the pressure of nitrogen from 0.2 torr to 0.6 torr. Further work is being carried out to characterize this behaviour.

Our findings have highlighted some key points about carrying out low temperature work in a low vacuum environment. These are;

- The temperature for onset of sublimation is higher than for high vacuum SEM

- The rate of any sublimation can be controlled as a function of chamber pressure

As a corollary to this last point, we have developed a methodology for introducing water vapour as an additional factor in stabilising frozen-hydrated samples at higher temperatures. This is described elsewhere [3]. 


\section{References}

[1] D. Tabor, Gases, liquids and solids, and other states of matter, $3^{\text {rd }}$ ed. Cambridge University press, Cambridge, 1991

[2] Gatan Alto 2100 Operators handbook

[3] D. Waller et al., elsewhere in these proceedings.

[4] The author would like to thank Unilever for their generous support

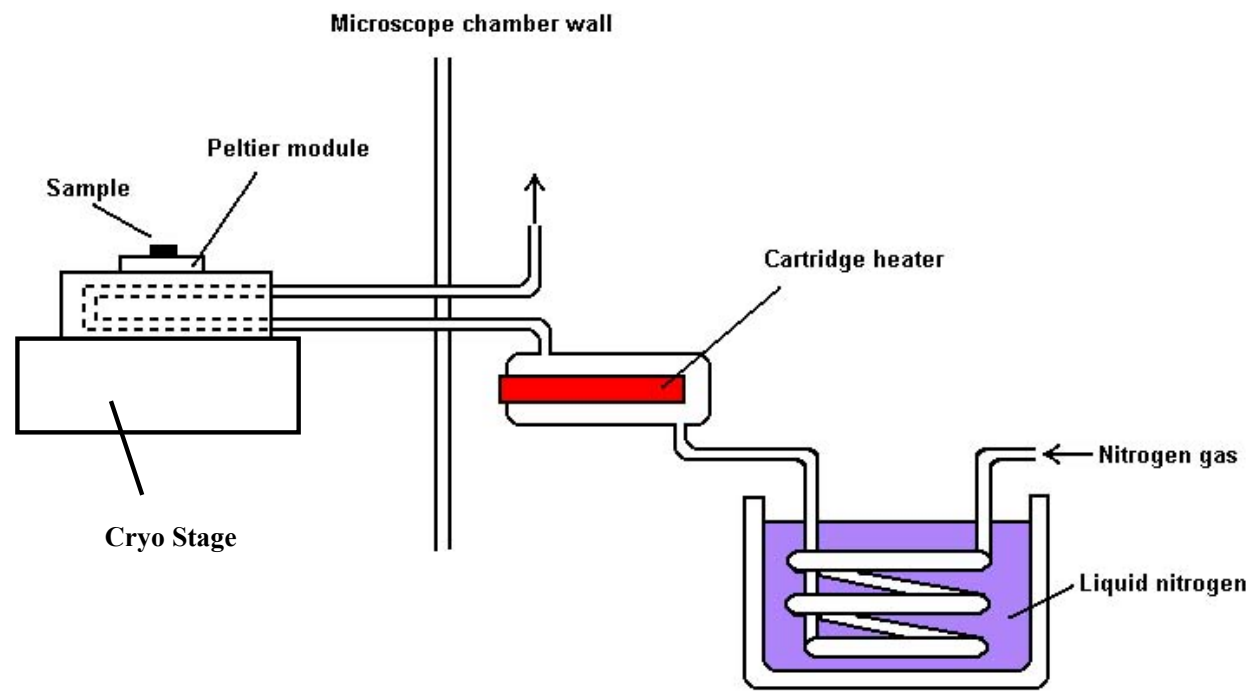

Figure 1. Low temperature ESEM apparatus.

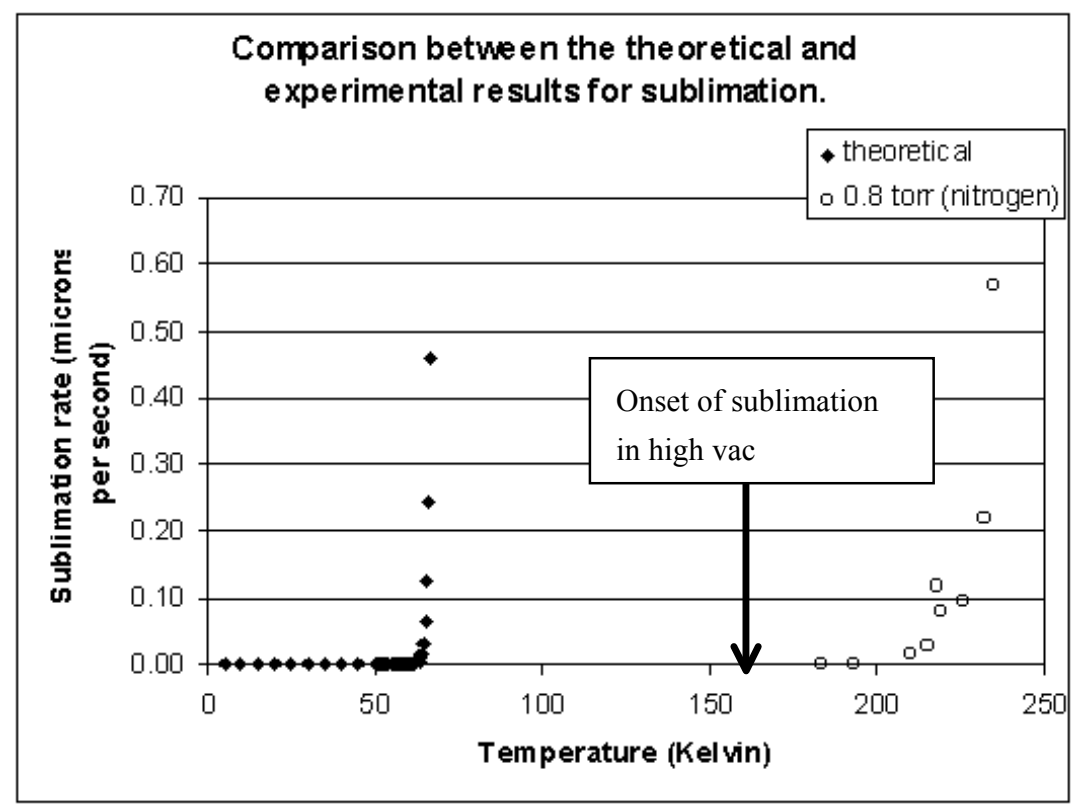

Figure 2. Experimental and theoretical sublimation rates 